Original Research Paper

\title{
Machine Motion Equations at the Internal Combustion Heat Engines
}

\author{
Florian Ion T. Petrescu \\ Department of the Theory of Mechanisms and Robots, Bucharest Polytechnic University, Bucharest, Romania
}

Article history

Received: 05-03-2015

Revised: 09-03-2015

Accepted: 06-04-2015

\section{Introduction}

In conditions which started to magnetic motors, oil fuel is decreasing, energy which was obtained by burning oil is replaced with nuclear energy, hydropower, solar energy, wind and other types of unconventional energy, in the conditions in which electric motors have been instead of internal combustion in public transport, but more recently they have entered in the cars world (Honda has produced a vehicle that uses a compact electric motor and electricity consumed by the battery is restored by a system that uses an electric generator with hydrogen combustion in cells, so we have a car that burns hydrogen, but has an electric motor), which is the role and prospects which have internal combustion engines type Otto, Diesel or Wankel (Amoresano et al., 2013;
Dawson, 2005; De Falco et al., 2013a; 2013b; Dieter, 2000; Eneşca, 2007; Ferguson and Kirkpatrick, 2001; Ganapathi and Robinson, 2013; Gunston, 1999; Gupta, 2006; Guzzella, 2004; Heywood, 1988; Karikalan et al., 2013; Lee, 2005; Liu, 1995; Mahalingam and Ramesh Bapu, 2013; Naima and Liazid, 2013; Narasiman et al., 2013; Petrescu and Petrescu, 2005; 2009a; 2009b; 2013a; 2013b; Petrescu et al., 2005; Petrescu and Petrescu, 2014; 2013c; 2013d; 2011; Petrescu, 2012a; 2012b; Piltan et al., 2012; Rahmani et al., 2013; Ravi and Subramanian, 2013; Ronney et al., 1994; Sapate and Tikekar, 2013; Sethusundaram et al., 2013; Zahari et al., 2013; Zhu et al., 2007)?

Internal combustion engines in four-stroke (Otto, Diesel, Wankel) are robust, dynamic, compact, powerful, reliable, economic, autonomous, independent and will be increasingly clean (Amoresano et al., 2013; Dawson, 
2005; De Falco et al., 2013a; 2013b; Dieter, 2000; Eneşca, 2007; Ferguson and Kirkpatrick, 2001; Ganapathi and Robinson, 2013; Gunston, 1999; Gupta, 2006; Guzzella, 2004; Heywood, 1988; Karikalan et al., 2013; Lee, 2005; Liu, 1995; Mahalingam and Ramesh Bapu, 2013; Naima and Liazid, 2013; Narasiman et al., 2013; Piltan et al., 2012; Rahmani et al., 2013; Ravi and Subramanian, 2013; Ronney et al., 1994; Sapate and Tikekar, 2013; Sethusundaram et al., 2013; Zahari et al., 2013; Zhu et al., 2007).

Magnetic motors (combined with the electromagnetic) are just in the beginning, but they offer us a good perspective, especially in the aeronautics industry (Gunston, 1999). Probably at the beginning they will not be used to act as a direct transmission, but will generate electricity that will fill the battery that will actually feed the engine (probably an electric motor).

The Otto engines or those with internal combustion in general, will have to adapt to hydrogen fuel (Eneşca, 2007). Hydrogen can be extracted industrially, practically from any item (or combination) through nuclear, chemical, by radiation, by burning, etc... (Most easily hydrogen can be extracted from water by breaking up into constituent elements, hydrogen and oxygen; by burning hydrogen one obtains water again that restores a circuit in nature, with no losses and no pollution) (Eneşca, 2007). Hydrogen must be stored in reservoirs cell (a honeycomb) for there is no danger of explosion; the best would be if we could breaking up water directly on the vehicle, in which case the reservoir would feed water (and there were announced a few successes) (Eneşca, 2007).

Now and the life of the jet engine begin to end (only in these forms). Even in these conditions internal combustion engines will be maintained in land vehicles (at least), for power, reliability and especially their dynamics.

Otto engines design (Amoresano et al., 2013; Dawson, 2005; De Falco et al., 2013a; 2013b; Dieter, 2000; Eneşca, 2007; Ferguson and Kirkpatrick, 2001; Ganapathi and Robinson, 2013; Gunston, 1999; Gupta, 2006; Guzzella, 2004; Heywood, 1988; Karikalan et al., 2013; Lee, 2005; Liu, 1995; Mahalingam and Ramesh Bapu, 2013; Naima and Liazid, 2013; Narasiman et al., 2013), includes and the dynamic design.

Old gasoline engines carry us every day for nearly 150 years. "Old Otto engine" (and his brother, Diesel) is today: Younger, more robust, more dynamic, more powerful, more economical, more independent, more reliable, quieter, cleaner, more compact, more sophisticated, more stylish, more secure and more especially necessary and wanted. At the global level we can manage to remove annually about 60,000 cars. But annually appear other million cars (Table 1).

\begin{tabular}{ll} 
Table 1. World cars produced & \\
\hline Year & Cars produced in the world \\
\hline 2011 & $59,929,016$ \\
2010 & $58,264,852$ \\
2009 & $47,772,598$ \\
2008 & $52,726,117$ \\
2007 & $53,201,346$ \\
2006 & $49,918,578$ \\
2005 & $46,862,978$ \\
2004 & $44,554,268$ \\
2003 & $41,968,666$ \\
2002 & $41,358,394$ \\
2001 & $39,825,888$ \\
2000 & $41,215,653$ \\
1999 & $39,759,847$ \\
\hline
\end{tabular}

In full energy crisis since 1970 until today, production and sale of cars equipped with internal combustion heat engines has skyrocketed, from some millions yearly to over sixty millions yearly now and the world fleet started from tens of millions reached today the billion. As long as we produce electricity and heat by burning fossil fuels is pointless to try to replace all thermal engines with electric motors, as loss of energy and pollution will be even larger. However, it is well to continuously improve the thermal engines, to reduce thus fuel consumption. Planet supports now about one billion motor vehicles in circulation. Even if we stop totally production of heat engines, would still need 10,000 years to eliminate total the existing car park in the current rate. Electric current is still produced in majority by combustion of hydrocarbons, making the hydrocarbon losses to be higher when we use electric motors. When we will have electric current obtained only from green energy or nuclear, sustainable and renewable energy sources, it is only then that we'll be able to enter gradually and electric motors (Piltan et al., 2012; Rahmani et al., 2013; Ravi and Subramanian, 2013; Ronney et al., 1994; Sapate and Tikekar, 2013; Sethusundaram et al., 2013; Zahari et al., 2013; Zhu et al., 2007).

Otto and diesel engines are today the best solution for the transport of our day-to-day work, together and with electric motors and those with reaction.

For these reasons it is imperative as we can calculate exactly the engine efficiency, in order to can increase it permanently.

\section{Nomenclature}

$J^{*}: \quad$ Is the moment of inertia (mass or mechanical) reduced to the motor shaft

$J_{\text {Max }}^{*}$ : Is the maximum moment of inertia (mass or mechanical) reduced to the motor shaft

$J_{\min }^{*}$ : Is the minimum moment of inertia (mass or mechanical) reduced to the motor shaft 
$J_{m}^{*}: \quad$ Is the average moment of inertia (mass or mechanical) reduced to the motor shaft

$v_{C}$ : Is the normal (cinematic) velocity of the point $C$

$v_{C}^{\text {Din.c }}:$ Is the dynamic velocity of the point $C$ (in compressor system)

$v_{C}^{\text {Din.m }}:$ Is the dynamic velocity of the point $C$ (in motor system)

$a_{C}$ : Is the normal (cinematic) acceleration of the point $C$

$a_{C}^{\text {Din.c }}:$ Is the dynamic acceleration of the point $C$ (in compressor system)

$a_{C}^{\text {Din.m }}:$ Is the dynamic acceleration of the point $C$ (in motor system)

$\varphi_{1}=\varphi: \quad$ Is the position angle of the crank

$\omega_{\mathrm{m}}=\omega_{\mathrm{n}}$ : Is the constant (nominal) angular rotation speed of the crank (the motor shaft)

$\omega: \quad$ Is the variable (dynamic) angular rotation speed of the crank (the motor shaft)

$\varepsilon$ : Is the angular rotation acceleration of the motor shaft; Appears only in dynamic schemes

$\varphi_{2},=\psi:$ Is the position angle of the rod (element 2), if the rod is considered from the point $C$

$\dot{\psi}: \quad$ Is the angular rotation speed of the rod (element 2)

$\varphi_{2}=\theta$ : Is the position angle of the rod, if the rod is considered from the point B

$1_{1}$ : $\quad$ Is the length of the crank

$1_{2}$ : $\quad$ Is the length of the rod (the connecting rod)

$\lambda: \quad$ Is the raport between $l_{1}$ and $l_{2}$

$D^{c}: \quad$ Is the dynamic coefficient (in compressor system)

$\dot{D}^{c}$ : $\quad$ Is the derivative of $D^{c}$ in function of the time

$D^{c}$ : $\quad$ Is the derivative of $D^{c}$ in function of the position angle of the motor shaft, $\varphi$

$D^{m}: \quad$ Is the dynamic coefficient (in motor system)

$\dot{D}^{m}$ : Is the derivative of $D^{m}$ in function of the time

$D^{m \prime}$ : Is the derivative of $D^{m}$ in function of the position angle of the motor shaft, $\varphi$

\section{Determining the First Machine Equation}

One presents the dynamic, original, machine motion equations. The equation of motion of the machine that generates angular speed of the shaft (which varies with position and rotation speed) is deduced by conservation kinetic energy of the machine. An additional variation of angular speed is added by multiplying by the coefficient dynamic (generated by the forces out of mechanism).

Kinetic energy conservation shows angular speed variation (from the main shaft) with inertial masses, while the dynamic coefficient introduces the variation of $\omega$ with forces acting in the mechanism (Petrescu and Petrescu, 2005; 2009a; 2009b; 2013a; 2013b;
Petrescu et al., 2005; Petrescu and Petrescu, 2014; 2013c; 2013d; 2011; Petrescu, 2012a; 2012b).

In system (1) one determines the variable rotation velocity of the (motor) shaft, in function of the position $\varphi$ of the shaft and of rotation nominal speed $\omega_{m}$. One starts from the equation of kinetics energy (that is conserved):

$$
\left\{\begin{array}{l}
\frac{1}{2} \cdot J_{m}^{*} \cdot \omega_{m}^{2}=\frac{1}{2} \cdot J_{\max }^{*} \cdot \omega_{\min }^{2}= \\
=\frac{1}{2} \cdot J_{\min }^{*} \cdot \omega_{\max }^{2}=\frac{1}{2} \cdot J^{*} \cdot \omega^{2} \Rightarrow \\
\Rightarrow J_{m}^{*} \cdot \omega_{m}^{2}=J_{\max }^{*} \cdot \omega_{\min }^{2}=J_{\min }^{*} \cdot \omega_{\max }^{2}=J^{*} \cdot \omega^{2} \\
\Rightarrow J_{m}^{*} \cdot \omega_{m}^{2}=J^{*} \cdot \omega^{2} \\
\omega^{2}=\frac{J_{m}^{*}}{J^{*}} \cdot \omega_{m}^{2} ; \omega=\sqrt{\frac{J_{m}^{*}}{J^{*}}} \cdot \omega_{m} \\
J_{m}^{*}=\frac{J_{\max }^{*}+J_{\min }^{*}}{2} \\
\omega_{m} \equiv \omega_{\text {med }} \equiv \omega_{n}=2 \cdot \pi \cdot v=2 \pi \cdot \frac{n}{60}=\frac{\pi}{30} \cdot n \\
\omega^{2}=\frac{J_{m}^{*}}{J^{*}} \cdot \omega_{m}^{2} \\
\omega=\sqrt{\frac{J_{m}^{*}}{J^{*}} \cdot \omega_{m}}
\end{array}\right.
$$

The first movement equation of the machine takes the initial forms (system 2) (were selected from system 1 just the two final relations):

$\left\{\begin{array}{l}\omega^{2}=\frac{J_{m}^{*}}{J^{*}} \cdot \omega_{m}^{2} \\ \omega=\sqrt{\frac{J_{m}^{*}}{J^{*}}} \cdot \omega_{m}\end{array}\right.$

Since $J^{*}$ is a function of the angle $\varphi$ and $\omega_{n}$ is a function of $n$, it follows that $\omega$ is a function of angle $\varphi$ and angular rotation speed $n=>\omega=\omega(\varphi, n)$.

An additional variation of angular speed is added by multiplying by the coefficient dynamic (generated by the forces out of mechanism). The final forms of the first movement equation of the machine can be seen in the system (3).

$$
\left\{\begin{array}{l}
\omega^{2}=\frac{J_{m}^{*}}{J^{*}} \cdot \omega_{m}^{2} \cdot D^{2} \\
\omega=\sqrt{\frac{J_{m}^{*}}{J^{*}}} \cdot \omega_{m} \cdot D
\end{array}\right.
$$

where, the dynamic coefficient $\mathrm{D}$ takes the values of the system (4) or (5); (4) when the engine works in 
compressor system and (5) when the mechanism works in motor system:

$$
\left\{\begin{array}{l}
D^{c}=\sin ^{2} \psi \\
\dot{D}^{c}=\sin 2 \psi \cdot \dot{\psi} \\
D^{c}=\sin 2 \psi \cdot \psi^{\prime} \\
\psi^{\prime}=\frac{\dot{\psi}}{\omega}
\end{array}\right.
$$

$$
\left\{\begin{array}{l}
D^{m}=\sin ^{2}(\psi-\phi) \\
\dot{D}^{m}=\sin 2(\psi-\phi) \cdot(\dot{\psi}-\omega) \\
D^{m \prime}=\sin [2(\psi-\phi)] \cdot\left(\psi^{\prime}-1\right)
\end{array}\right.
$$

Method is applied separately for two distinct situations: When the engine is working on a compressor and into the motor system. Calculations should be made for an engine with a single cylinder (Petrescu and Petrescu, 2005; 2009a; 2009b; 2013a; 2013b; Petrescu et al., 2005; Petrescu and Petrescu, 2014; 2013c; 2013d; 2011; Petrescu, 2012a; 2012b).

\section{Determining the Second Machine Equation}

The second machine equation is determined by the derivation of the first machine equation in function of the time (6):

$$
\left\{\begin{array}{l}
\omega^{2}=\frac{J_{m}^{*}}{J^{*}} \cdot \omega_{m}^{2} \cdot D^{2} \Rightarrow \\
\Rightarrow \omega^{2} \cdot J^{*}=J_{m}^{*} \cdot \omega_{m}^{2} \cdot D^{2} \\
\frac{d}{d t}\left(\omega^{2} \cdot J^{*}=J_{m}^{*} \cdot \omega_{m}^{2} \cdot D^{2}\right) \Rightarrow \\
2 \cdot \omega \cdot \frac{d \omega}{d t} \cdot J^{*}+\omega^{2} \cdot \frac{d J^{*}}{d t}=J_{m}^{*} \cdot \omega_{m}^{2} \cdot 2 \cdot D \cdot \frac{d D}{d t} \Rightarrow \\
\Rightarrow 2 \cdot \omega \cdot \frac{d \omega}{d t} \cdot J^{*}+\omega^{2} \cdot \frac{d J^{*}}{d \phi} \cdot \frac{d \phi}{d t}= \\
=2 \cdot J_{m}^{*} \cdot \omega_{m}^{2} \cdot D \cdot \frac{d D}{d \phi} \cdot \frac{d \phi}{d t} \Rightarrow \\
\Rightarrow \omega \cdot \varepsilon \cdot J^{*}+\frac{1}{2} \omega^{2} \cdot \frac{d J^{*}}{d \phi} \cdot \omega=J_{m}^{*} \cdot \omega_{m}^{2} \cdot D \cdot \frac{d D}{d \phi} \cdot \omega \\
\Rightarrow \varepsilon \cdot J^{*}+\frac{1}{2} \omega^{2} \cdot \frac{d J^{*}}{d \phi}=\frac{1}{2} \omega^{2} \cdot J_{m}^{* \prime}=\omega_{m}^{2} \cdot D \cdot \frac{d D}{d \phi} \Rightarrow \\
\varepsilon=-\frac{1}{2} \omega^{2} \cdot \frac{J^{* \prime}}{J^{*}}+\frac{J_{m}^{*}}{J^{*}} \cdot \omega_{m}^{2} \cdot D \cdot D \cdot D \cdot D^{\prime} \\
\varepsilon=-\frac{1}{2} \frac{J_{m}^{*} \cdot J^{* \prime}}{J^{* 2}} \cdot \omega_{m}^{2} \cdot D^{2}+\frac{J_{m}^{*}}{J^{*}} \cdot \omega_{m}^{2} \cdot D \cdot D^{\prime}
\end{array}\right.
$$

One keeps just the final relations (7):

$$
\left\{\begin{array}{l}
\varepsilon \cdot J^{*}+\frac{1}{2} \omega^{2} \cdot J^{* \prime}=J_{m}^{*} \cdot \omega_{m}^{2} \cdot D \cdot D^{\prime} \\
\varepsilon=-\frac{1}{2} \omega^{2} \cdot \frac{J^{* \prime}}{J^{*}}+\frac{J_{m}^{*}}{J^{*}} \cdot \omega_{m}^{2} \cdot D \cdot D^{\prime} \\
\varepsilon=-\frac{1}{2} \frac{J_{m}^{*} \cdot J^{* \prime}}{J^{* 2}} \cdot \omega_{m}^{2} \cdot D^{2}+\frac{J_{m}^{*}}{J^{*}} \cdot \omega_{m}^{2} \cdot D \cdot D^{\prime}
\end{array}\right.
$$

\section{Observation}

If one compares the presented equation of motion of the machine (6-7) with the fever (Lagrange), it can identify the reduced (rotation) force, (the reduced load: 8):

$$
\left\{\begin{array}{l}
\left\{\begin{array}{l}
\varepsilon \cdot J^{*}+\frac{1}{2} \omega^{2} \cdot J^{* \prime}=J_{m}^{*} \cdot \omega_{m}^{2} \cdot D \cdot D^{\prime} \\
\varepsilon \cdot J^{*}+\frac{1}{2} \omega^{2} \cdot J^{* \prime}=M^{*}
\end{array}\right. \\
\Rightarrow M^{*}=J_{m}^{*} \cdot \omega_{m}^{2} \cdot D \cdot D^{\prime}
\end{array} \Rightarrow\right.
$$

\section{Application to the Otto Engine}

To determine dynamics at an Otto engine must (first at all) to set the formula of reduced moment of inertia (9); Fig. 1 (it takes $a_{1}=0$ ).

$$
\left\{\begin{array}{l}
J^{*}=J_{G_{1}}+J_{G_{2}} \cdot\left(\frac{\dot{\psi}}{\omega}\right)^{2}+m_{3} \cdot\left(\frac{v_{G_{3}}}{\omega}\right)^{2}+m_{2} \cdot\left(\frac{v_{G_{2}}}{\omega}\right) \\
=J_{G_{1}}+J_{G_{2}} \cdot \lambda^{2} \cdot \frac{\sin ^{2} \phi}{\sin ^{2} \psi}+m_{3} \cdot r^{2} \cdot \frac{\sin ^{2}(\psi-\phi)}{\sin ^{2} \psi} \\
+m_{2} \cdot\left(r^{2}+a^{2} \cdot \psi^{\prime 2}-2 a r \cdot \psi^{\prime} \cdot \cos (\psi-\phi)\right) \\
=J_{G_{1}}+J_{G_{2}} \cdot \lambda^{2} \cdot \frac{\sin ^{2} \phi}{\sin ^{2} \psi}+m_{3} \cdot r^{2} \cdot \frac{\sin ^{2}(\psi-\phi)}{\sin ^{2} \psi} \\
+m_{2} \cdot\left(r^{2}+a^{2} \cdot \lambda^{2} \cdot \frac{\sin ^{2} \phi}{\sin ^{2} \psi}-2 a \cdot r \cdot \lambda \cdot \frac{\sin \phi}{\sin \psi} \cdot \cos (\psi-\phi)\right. \\
J^{*}=J_{G_{1}}+m_{2} \cdot r^{2}+\left(J_{G_{2}}+m_{2} \cdot a{ }^{2}\right) \cdot \lambda^{2} \cdot \frac{\sin ^{2} \phi}{\sin { }^{2} \psi} \\
+m_{3} \cdot r^{2} \cdot \frac{\sin ^{2}(\psi-\phi)}{\sin ^{2} \psi}-2 m_{2} \cdot a \cdot r \cdot \lambda \cdot \frac{\sin \phi \cdot \cos (\psi-\phi)}{\sin \psi}
\end{array}\right.
$$

One determines:

$$
J_{\text {Max }}^{*}, J_{\min }^{*}, J_{m e d}^{*} \equiv J_{m}^{*}=\frac{J_{M a x}^{*}+J_{\min }^{*}}{2}
$$

And $J^{*}$, with relation (10): 


$$
\left\{\begin{array}{l}
J^{* \prime}=\frac{1}{\sin ^{4} \psi} \cdot\left\{\left(J_{G_{2}}+m_{2} \cdot a^{2}\right) \cdot \lambda^{2}\right. \\
\cdot\left(\sin 2 \phi \cdot \sin ^{2} \psi-2 \lambda \cdot \sin ^{3} \phi \cdot \cos \psi\right) \\
+m_{3} \cdot r^{2} \cdot\left[\sin 2(\psi-\phi) \cdot\left(\lambda \sin \phi \cdot \sin \psi-\sin ^{2} \psi\right)\right. \\
\left.-2 \lambda \cdot \sin ^{2}(\psi-\phi) \cdot \cos \psi \cdot \sin \phi\right] \\
+2 m_{2} a r \lambda \cdot[\sin \phi \cdot \sin \psi \cdot \sin (\psi-\phi) \\
\cdot\left(\lambda \sin \phi \cdot \sin \psi-\sin ^{2} \psi\right) \\
+\lambda \cdot \sin ^{2} \phi \cdot \sin \psi \cdot \cos (\psi-\phi) \cdot \cos \psi \\
\left.\left.-\sin ^{3} \psi \cdot \cos \phi \cdot \cos (\psi-\phi)\right]\right\}
\end{array}\right.
$$

How to work: With $J^{*}, J^{* \prime}$ and $J_{m}^{*}$ it determines the variable $\omega$ and $\varepsilon$ with relations $(3,7)$, or $(11)$ :

$$
\left\{\begin{array}{l}
\omega=\sqrt{\frac{J_{m}^{*}}{J^{*}}} \cdot \omega_{m} \cdot D \\
\varepsilon=-\frac{1}{2} \frac{J_{m}^{*} \cdot J^{* \prime}}{J^{* 2}} \cdot \omega_{m}^{2} \cdot D^{2}+\frac{J_{m}^{*}}{J^{*}} \cdot \omega_{m}^{2} \cdot D \cdot D^{\prime}
\end{array}\right.
$$

Then one calculates the dynamic cinematic with the relations from the system (12):

$$
\left\{\begin{array}{l}
y_{C}=r \cdot \sin \phi-l \cdot \sin \psi \\
\dot{\psi}=\lambda \cdot \frac{\sin \phi}{\sin \psi} \cdot \omega \\
v_{C}^{D i n} \equiv \dot{y}_{C}=r \cdot \cos \phi \cdot \omega-l \cdot \cos \psi \cdot \dot{\psi} \\
\ddot{\psi}=\frac{\lambda \cos \phi \cdot \sin ^{2} \psi-\lambda^{2} \sin ^{2} \phi \cdot \cos \psi}{\sin ^{3} \psi} \cdot \omega^{2}+\frac{\lambda \sin \phi}{\sin \psi} \cdot \varepsilon \\
a_{C}^{D i n} \equiv \ddot{y}_{C}=-r \cdot \sin \phi \cdot \omega^{2}+r \cdot \cos \phi \cdot \varepsilon \\
+\frac{l \cdot \lambda^{2} \cdot \sin ^{2} \phi}{\sin \psi} \cdot \omega^{2}-l \cdot \cos \psi \cdot \ddot{\psi}
\end{array}\right.
$$

\section{Dynamic Kinematics Analysis for the Otto Engine in Compressor System}

Now, one can see the engine main mechanism in compressor system (when the motor mechanism is acting from the crank) (Petrescu and Petrescu, 2005; 2009a; 2009b; 2013a; 2013b; Petrescu et al., 2005; Petrescu and Petrescu, 2014; 2013c; 2013d; 2011; Petrescu, 2012a; $2012 \mathrm{~b}$ ). It is determining now, the velocities and the accelerations of the piston and motor shaft, normal and dynamic (Fig. 2-5).

\section{Dynamic Kinematics Analysis for the Otto Engine in Motor System}

Now, one can see the engine main mechanism in motor system (when the motor mechanism is acting from the piston) (Petrescu and Petrescu, 2005; 2009a; 2009b; 2013a; 2013b; Petrescu et al., 2005; Petrescu and Petrescu, 2014; 2013c; 2013d; 2011; Petrescu, 2012a; $2012 \mathrm{~b}$ ). It is determining now, the velocities and the accelerations of the piston and motor shaft, normal and dynamic (Fig. 6-9).

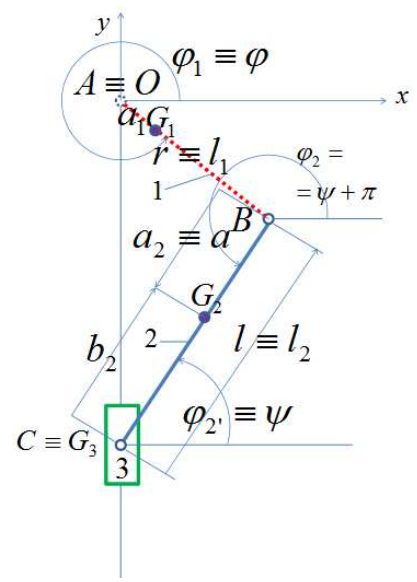

Fig. 1. The geometry of an Otto engine mechanism

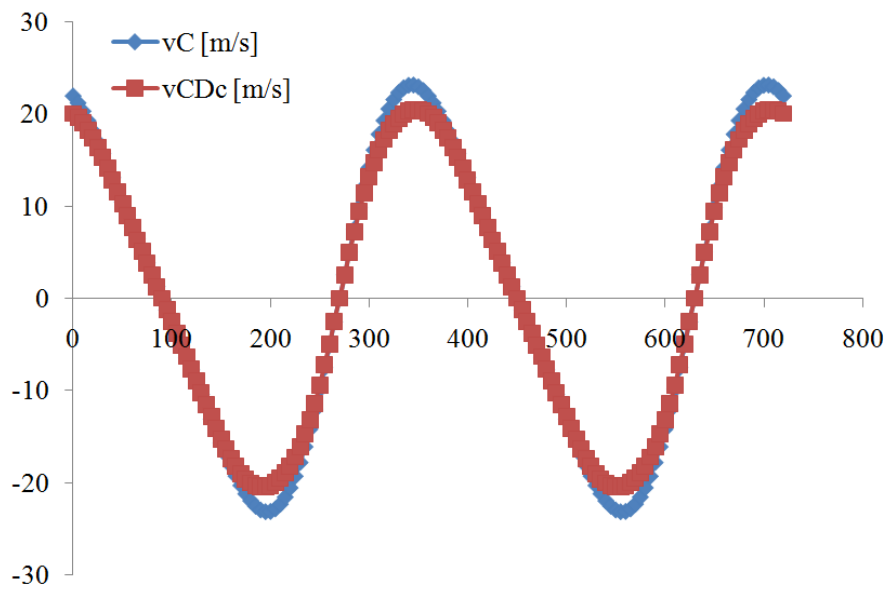

Fig. 2. The velocities of the piston, when the engine works in the compressor system 


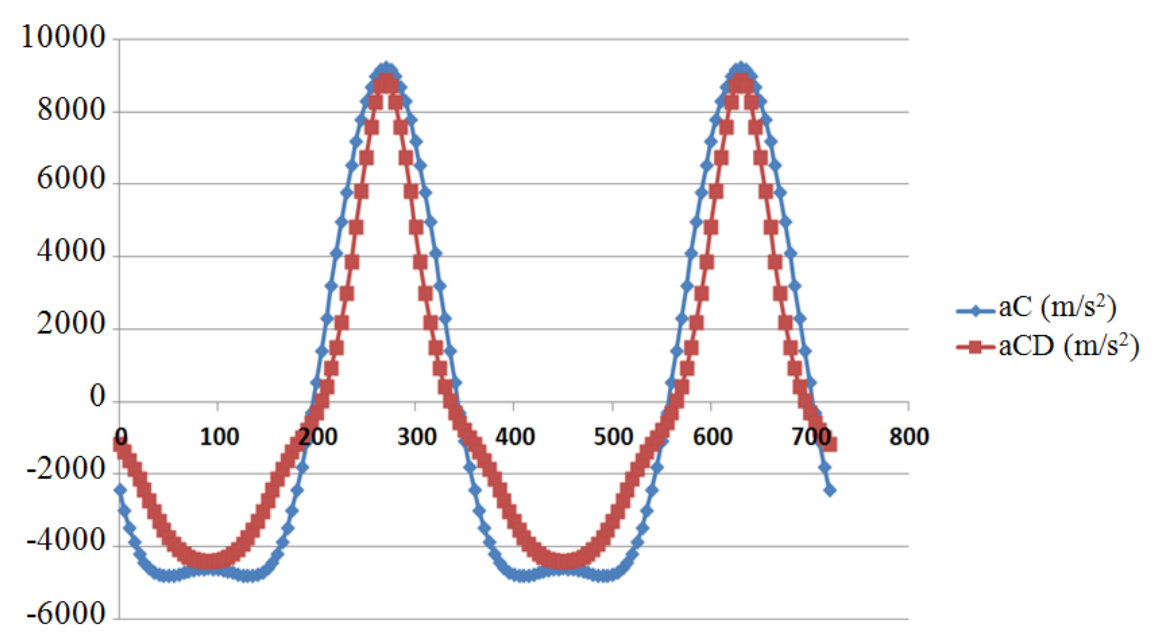

Fig. 3. The accelerations of the piston, when the engine works in the compressor system

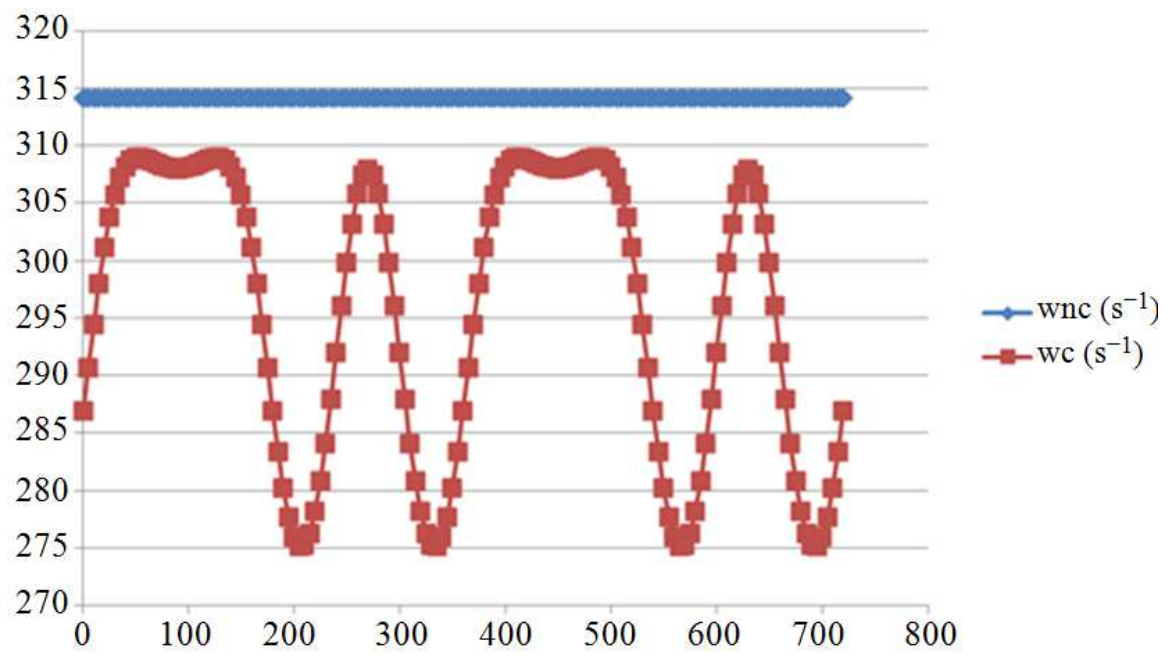

Fig. 4. The angular velocities of the motor shaft, when the engine works in the compressor system

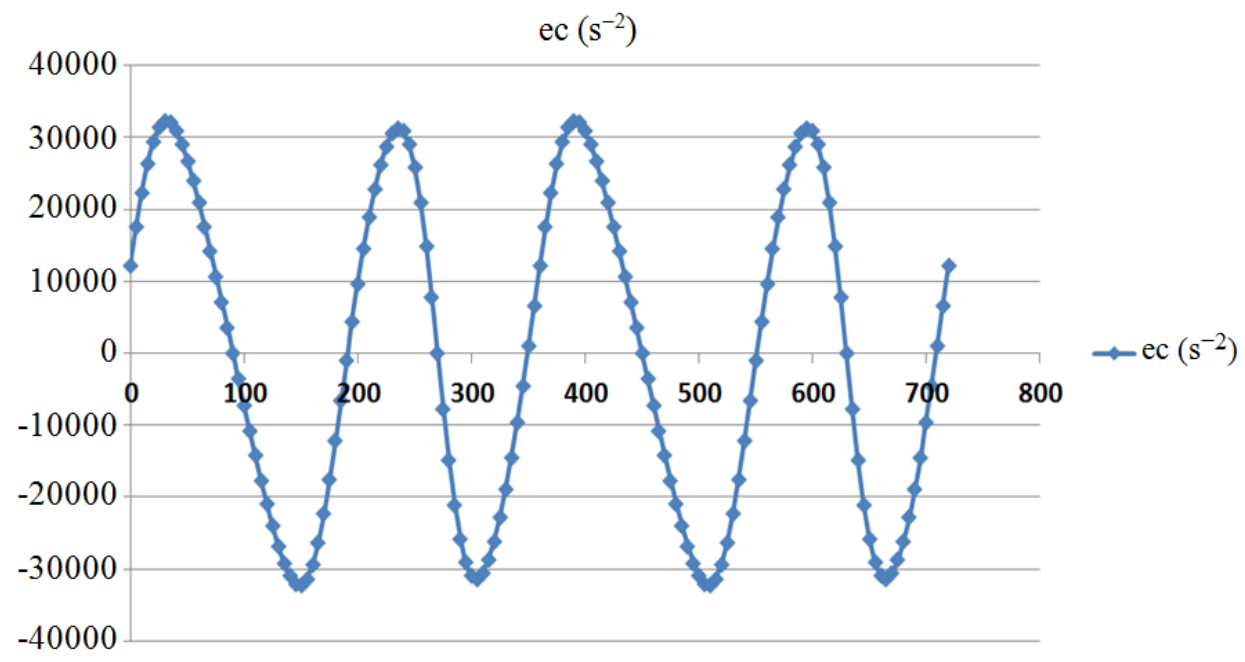

Fig. 5. The angular accelerations of the motor shaft, when the engine works in the compressor system 


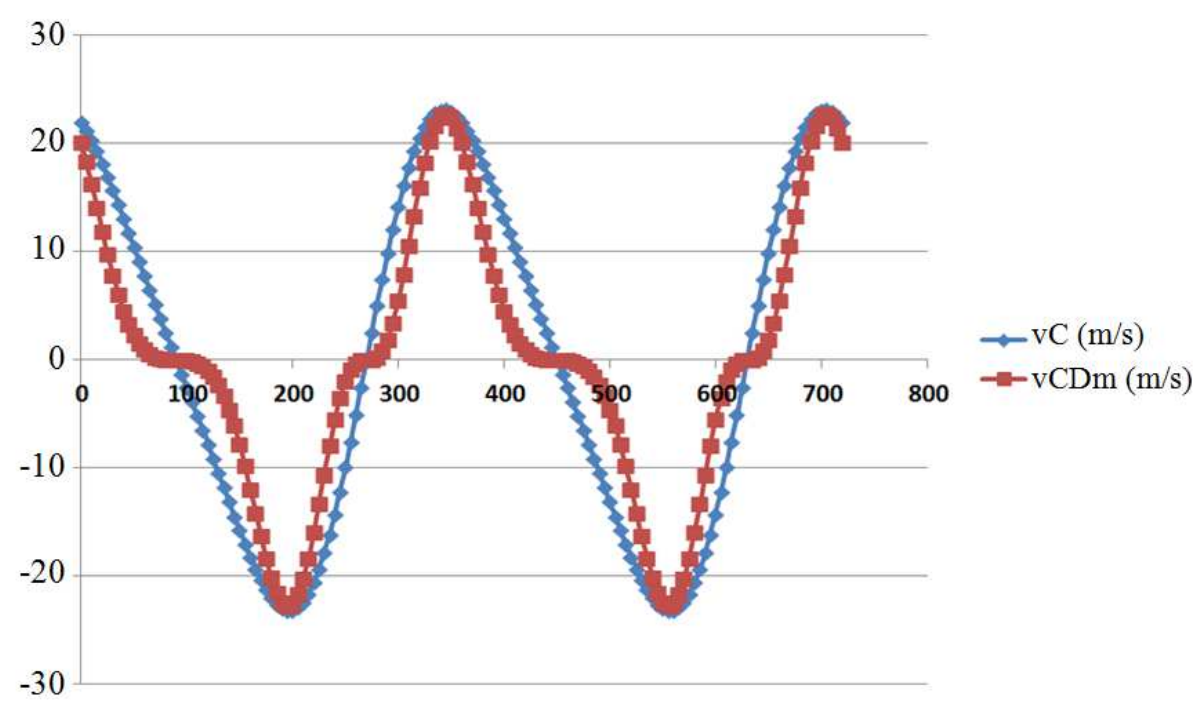

Fig. 6. The velocities of the piston, when the engine works in the motor system

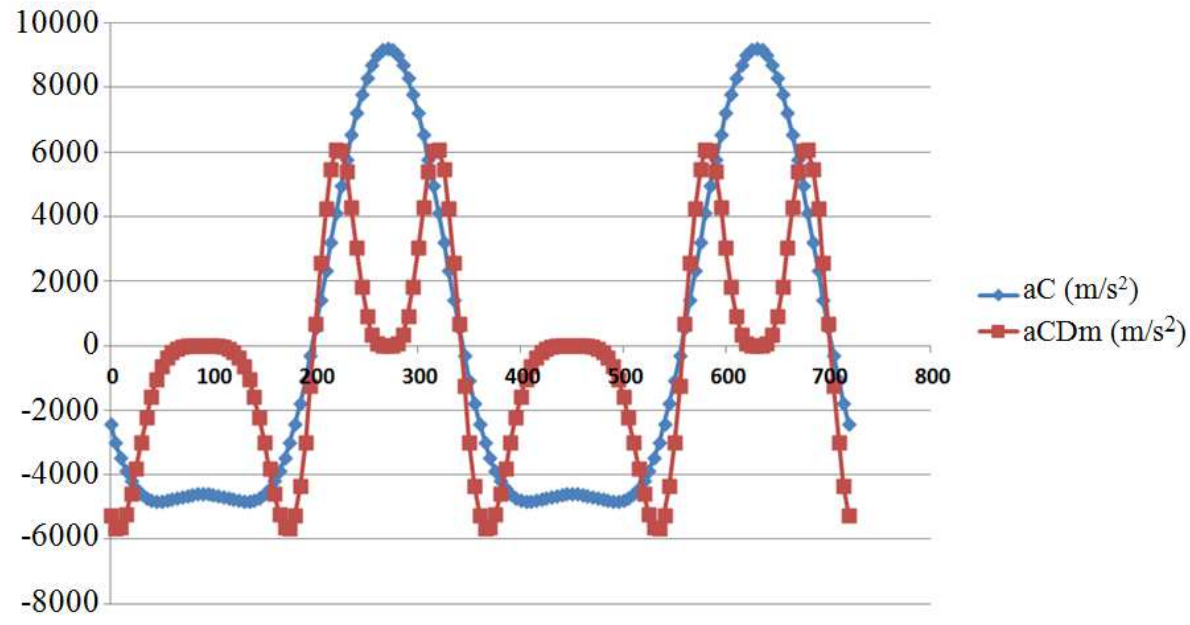

Fig. 7. The accelerations of the piston, when the engine works in the motor system

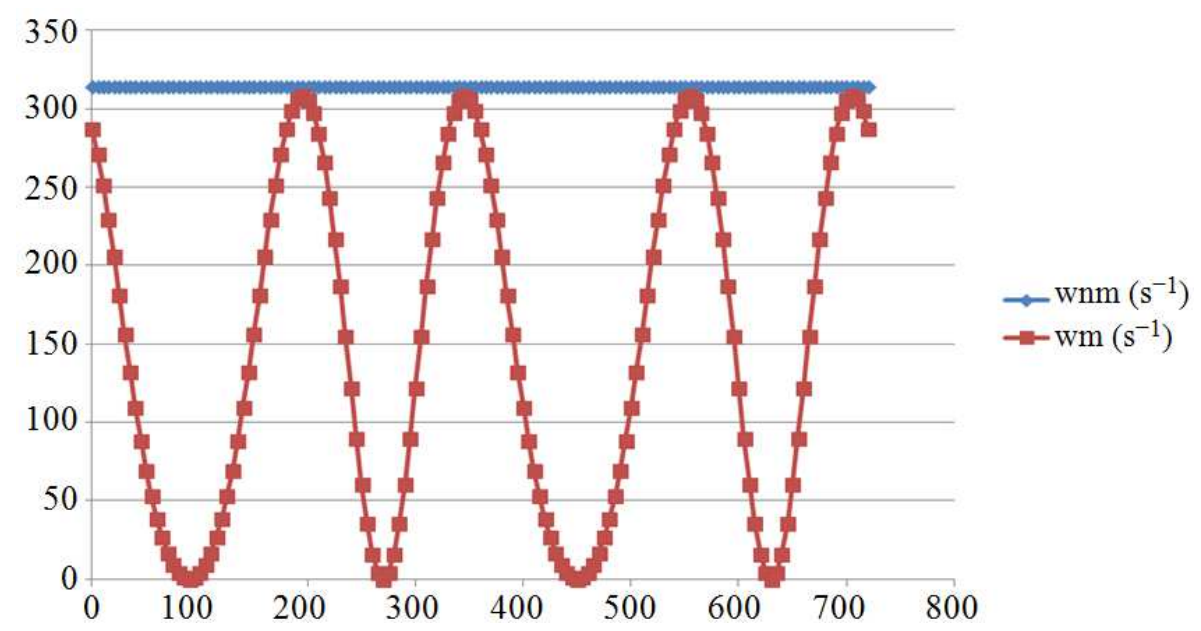

Fig. 8. The angular velocities of the motor shaft, when the engine works in the motor system 


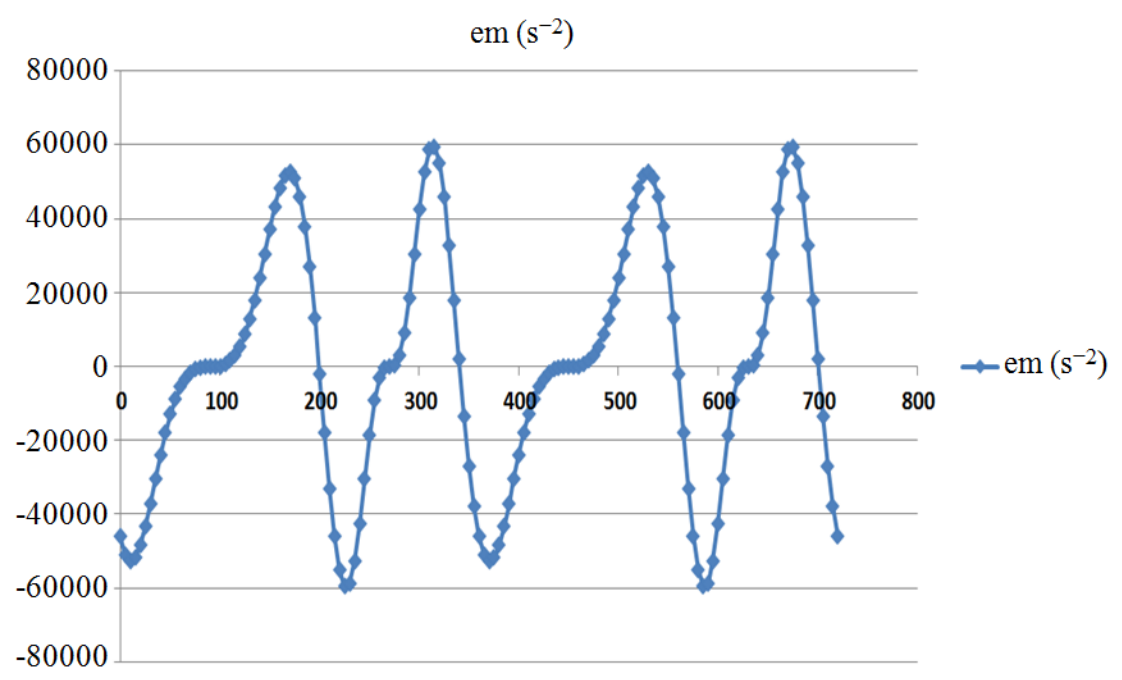

Fig. 9. The angular accelerations of the motor shaft, when the engine works in the motor system

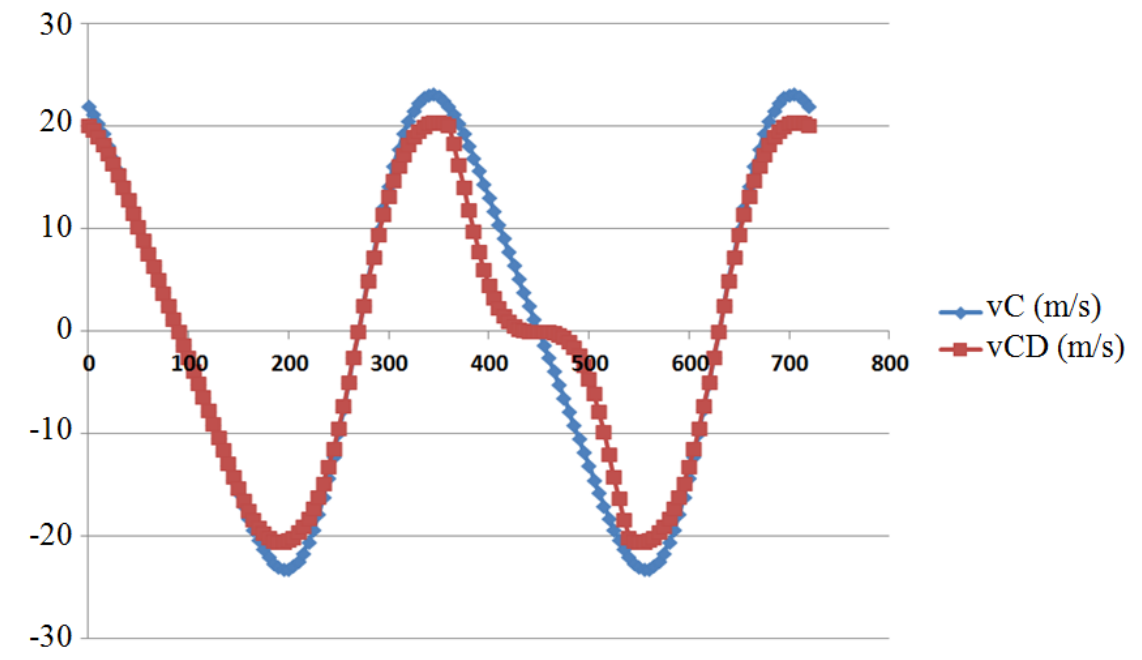

Fig. 10. The velocities of the piston, for a mono cylinder engine

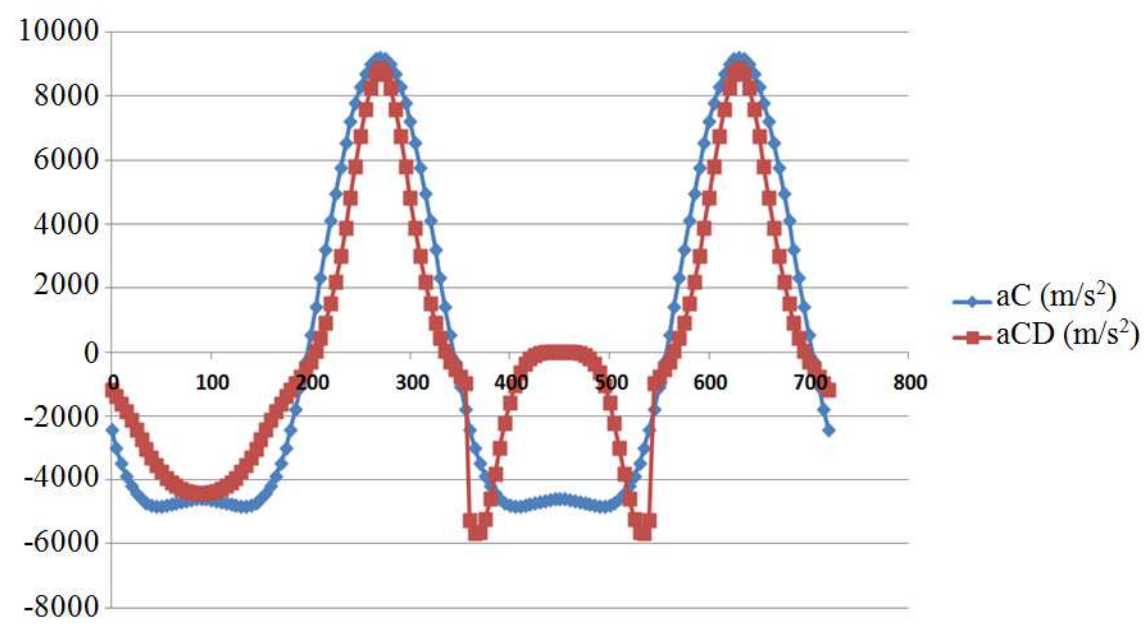

Fig. 11. The accelerations of the piston, for a mono cylinder engine, oriented upside down 


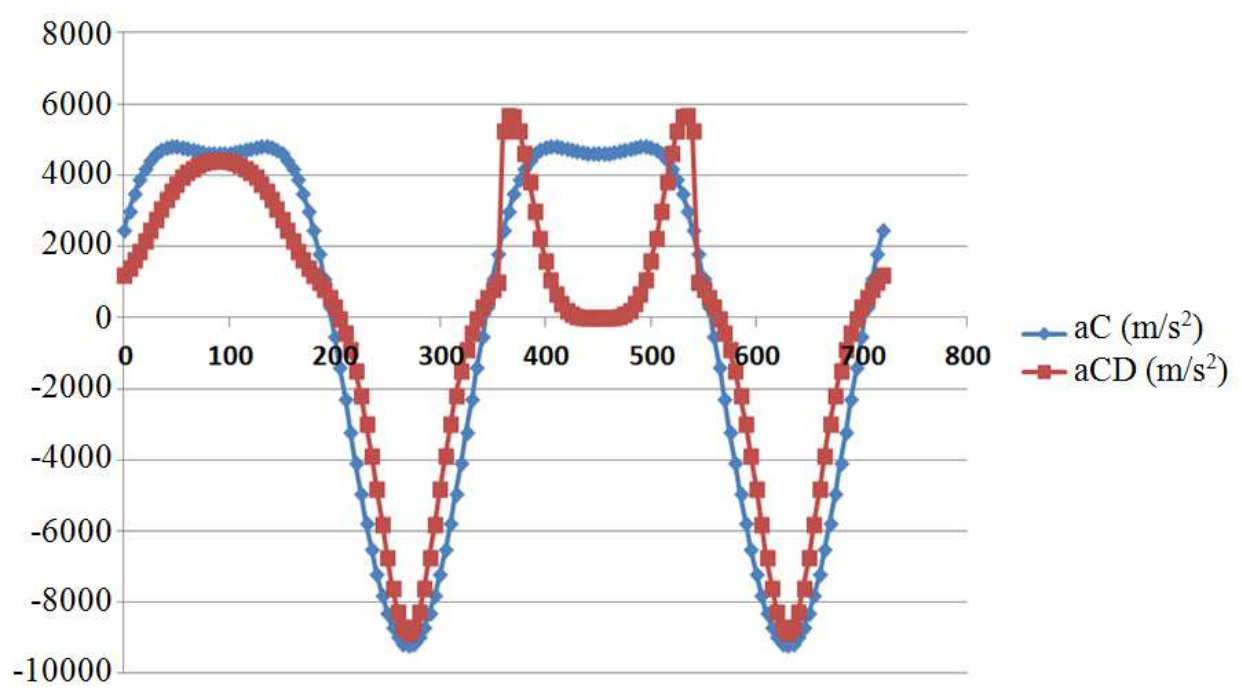

Fig. 12. The accelerations of the piston, for a mono cylinder engine, oriented head upward

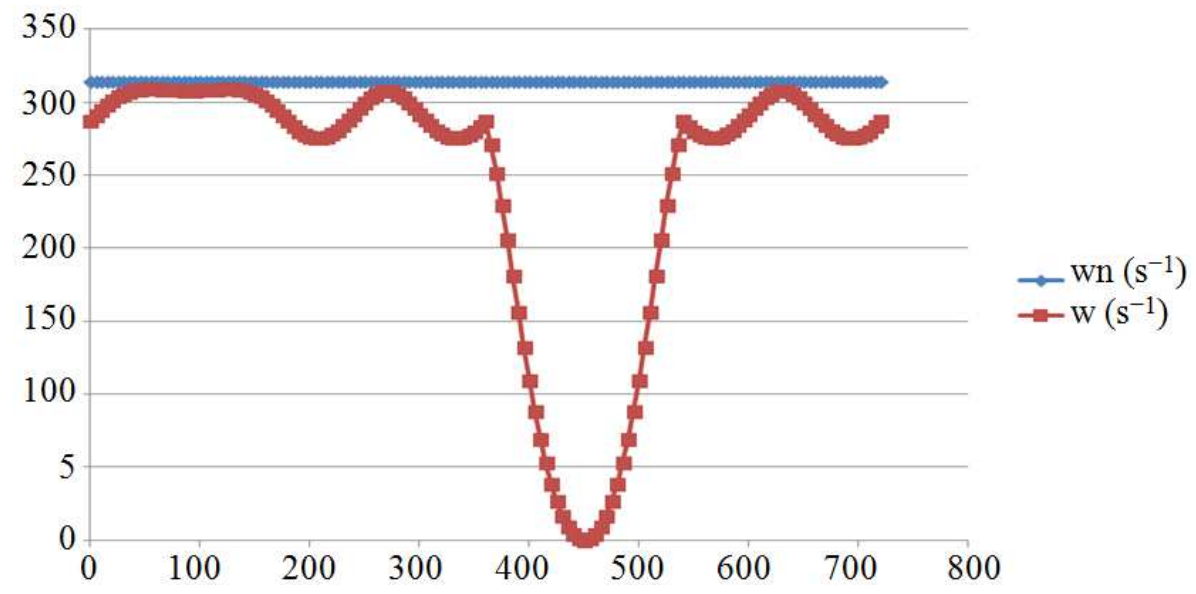

Fig. 13. The angular velocities of the motor shaft, for a mono cylinder engine

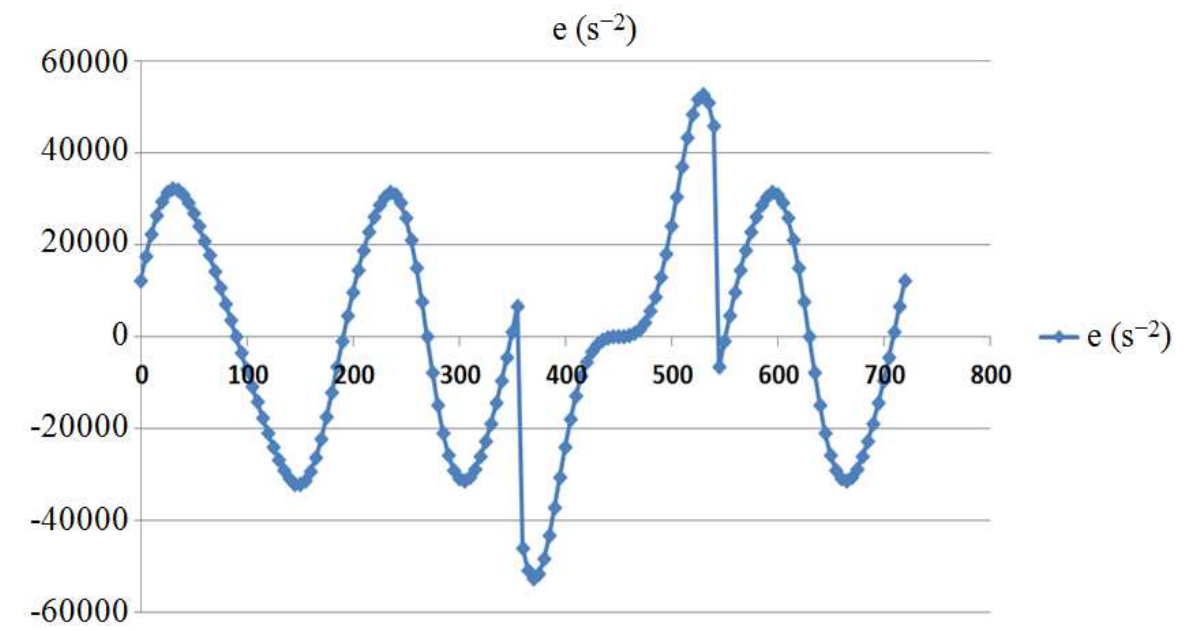

Fig. 14. The angular accelerations of the motor shaft, for a mono cylinder engine 


\section{Dynamic Kinematics Analysis for an Otto Engine, Mono-cylinder}

Now, one can see the engine main mechanism in motor and compressor system (when the motor mechanism is acting from the piston and from the crank). It is determining now, the velocities and the accelerations of the piston and motor shaft, normal and dynamic (Fig. 10-14).

\section{Discussion}

In full energy crisis since 1970 until today, production and sale of cars equipped with internal combustion heat engines has skyrocketed, from some millions yearly to over sixty millions yearly now and the world fleet started from tens of millions reached today the billion. As long as we produce electricity and heat by burning fossil fuels is pointless to try to replace all thermal engines with electric motors, as loss of energy and pollution will be even larger. However, it is well to continuously improve the thermal engines, to reduce thus fuel consumption. Planet supports now about one billion motor vehicles in circulation. Even if we stop totally production of heat engines, would still need 10,000 years to eliminate total the existing car park in the current rate. Otto and diesel engines are today the best solution for the transport of our day-to-day work, together and with electric motors and those with reaction. For these reasons it is imperative as we can calculate exactly the engine efficiency, in order to can increase it permanently. However, it is well to continuously improve the thermal engines, to reduce thus fuel consumption.

\section{Conclusion}

To the internal combustion heat engines the real velocities and accelerations (in dynamic regimes) are different that the cinematic (classic) velocities and accelerations. In this study one can see the engine main mechanism in motor and compressor system (when the motor mechanism is acting from the piston and from the crank). It is determining now, the velocities and the accelerations of the piston and motor shaft, normal and dynamic.

The first movement equation of the machine takes the initial forms (see the relations 2).

The second machine equation is determined by the derivation of the first machine equation in function of the time (see the relations from the system 6).

\section{Acknowledgment}

Research contract: Contract number 36-5-4D/1986 from 24IV1985, beneficiary CNST RO (Romanian
National Center for Science and Technology) improving dynamic mechanisms internal combustion engines.

\section{Ethics}

Author declares that are not ethical issues that may arise after the publication of this manuscript.

\section{References}

Amoresano, A., V. Avagliano, V. Niola and G. Quaremba, 2013. The assessment of the in-cylinder pressure by means of the morpho-dynamical vibration analysis-methodology and application. IREME, 7: 999-1006.

Dawson, J., 2005. An experimental and computational study of internal combustion engine modeling for controls oriented research. Ph.D. Thesis, Ohio State University.

De Falco, D., G. Di Massa, S. and S. Strano, 2013 a. Motorcycle handlebar dynamic response: Theoretical and experimental investigation. IREME, 7: 795-801.

De Falco, D., G. Di Massa and S. Pagano, 2013b. A full scale motorcycle dynamic rig. IREME, 7: 519-526.

Dieter, G., 2000. Engineering Design. 1st Edn., McGraw Hill, Boston.

Eneşca, I.A., 2007. Noi materiale nanostructurate pentru tehnologia hidrogenului. Ph.D. Thesis, Transilvania University of Braşov, Braşov, Romania.

Ferguson, C.R. and A.T. Kirkpatrick, 2001. Internal Combustion Engines: Applied Thermosciences. 2nd Edn., John Wiley and Sons, Inc., New York, ISBN-10: 0471356174, pp: 369.

Ganapathi, P. and Y. Robinson, 2013. Experimental investigation on the performance, emission and combustion characteristics of a diesel engine fuelled with polymer oil-ethanol blends. IREME, 7: 919-924.

Gunston, B., 1999. Development of Piston Aero Engines. 2nd Edn., Patrick Stephens Ltd., Sparkford, UK., ISBN-10: 0-7509-4478-1, pp: 21.

Gupta, H.N., 2006. Fundamentals of Internal Combustion Engines. 1st Edn., Prentice-Hall, New Delhi, ISBN-10: 812032854X, pp: 616.

Guzzella, L., 2004. Introduction to Modeling and Control of Internal Combustion Engine Systems. 1st Edn., Springer Science and Business Media, New York, ISBN-10: 354022274X, pp: 300.

Heywood, J., 1988. Internal Combustion Engine Fundamentals. 1st Edn., McGraw-Hill, New York, ISBN-10: 007028637X, pp: 930.

Karikalan, L., M. Chandrasekaran and K. Sudhagar, 2013. Comparative studies on vegetable oil usage in C.I engines as an alternative to diesel fuel. IREME, 7: 705-715. 
Lee, B., 2005. Methodology for rapid static and dynamic model-based engine calibration and optimization. Ph.D. Thesis, The Ohio State University.

Liu, T.H., 1995. A maximum torque control with a controlled capacitor for a single-phase induction motor. IEEE Trans. Indust. Electr. 42: 17-24. DOI: $10.1109 / 41.345841$

Mahalingam, S. and B.R. Ramesh Bapu, 2013. Experimental and emission analysis of rubber seed oil and jatropha oil blends with diesel in compression ignition engine. IREME, 7: 955-959.

Naima, K. and A. Liazid, 2013. Numerical investigation on combustion behaviors of direct-injection spark ignition engine fueled with CNG-hydrogen blends. IREME, 7: 652-663.

Narasiman, V., S. Jeyakumar and M. Mani, 2013. Optimizing the compression ratio of C.I engine fuelled in sardine oil ethyl ester. IREME, 7: 463-467.

Petrescu, R.V. and F.I. Petrescu, 2005. Determining the mechanical efficiency of Otto engine's mechanism. Proceedings of the International Symposium on Theory of Machines and Mechanisms, (TMM' 05), Bucharest, pp: 141-146.

Petrescu, F.I., R.V. Petrescu and N. Popescu, 2005. The efficiency of cams. Proceedings of the 2nd International Conference "Mechanics and Machine Elements, Nov. 4-6, Technical University of Sofia, Sofia, Bulgaria, pp: 237-243.

Petrescu, R.V. and F.I. Petrescu, 2009a. Otto engine design. Acta Technica Napocensis, Series: Applied Mathematics and Mechanics.

Petrescu, F.I. and R.V. Petrescu, 2009b. V engine design. Acta Technica Napocensis, Series: Applied Mathematics and Mechanics.

Petrescu, F.I. and R.V. Petrescu, 2011. Dinamica Mecanismelor de Distributie. 1st Edn., Create Space Publisher, USA., ISBN-10: 1468052659, pp: 188.

Petrescu, F.I., 2012a. Bazele Analizei si Optimizarii Sistemelor cu Memorie Rigida-Color: Curs si Aplicatii. Create Space Publisher, USA, ISBN-10: 1475065892, pp: 164.

Petrescu, F.I., 2012b. Teoria Mecanismelor-Curs si Aplicatii. 1st Edn., Create Space Publisher, USA, ISBN-10: 978-1-4792-9362-9, pp: 284.
Petrescu, F.I. and R.V. Petrescu, 2013a. Forces and efficiency of cams. IREME, 7: 507-511.

Petrescu, F.I. and R.V. Petrescu, 2013b. Cams with high efficiency. IREME, 7: 599-606.

Petrescu, F.I. and R.V. Petrescu, 2013c. An algorithm for setting the dynamic parameters of the classic distribution mechanism. IREMOS, 6: 1637-1641.

Petrescu, F.I. and R.V. Petrescu, 2013d. Dynamic Synthesis of the rotary cam and translated tappet with roll. IREMOS J., 6: 600-607.

Petrescu, F.I. and R.V. Petrescu, 2014. Cam gears dynamics in the classic distribution. Independent $\mathrm{J}$. Manage. Product., 5: 166-185.

Piltan, F., M. Piran, M. Akbari and M. Barzegar, 2012. Baseline tuning methodology supervisory sliding mode methodology: Applied to IC engine. Int. J. Adv. Applied Sci., 1: 116-124. e

Rahmani, L., B. Draoui, M. Bouanini and E. Benachour, 2013. CFD study on heat transfer to bingham fluid during with gate impeller. IREME, 7: 1074-1079.

Ravi, S. and R. Subramanian, 2013. Diesel fuel additives: An overview. IREME, 7: 698-704.

Ronney, P.D., M. Shoda, S.T. Waida and E.J. Durbin, 1994. Throttleless premixed-charge engines: Concept and experiment. J. Automobile Eng., 208: 13-24. DOI: 10.1243/PIME_PROC_1994_208_153_02

Sapate, K.D. and A.N. Tikekar, 2013. Engine mapping for improvement in fuel efficiency of two stroke SI engine. IREME, 7: 392-394.

Sethusundaram, P.P., K.P. Arulshri and K. Mylsamy, 2013. Biodiesel blend, fuel properties and its emission characteristics sterculia oil in diesel engine. IREME, 7: 925-929.

Zahari, I., M.A. Abras, N.I. Mat Arishad, S.F. Zainal and M.F. Muhamad, 2013. Experimental study to identify common engine part load conditions between Malaysian city driving and NEDC test. IREME, 7: 1152-1158.

Zhu, G., I. Haskara and J. Winkelman, 2007. Closed-loop ignition timing control for SI engines using ionization current feedback. IEEE Trans. Control Syst., 15: 416-427. DOI: 10.1109/TCST.2007.894634 